\title{
Combining Pilot-Symbol-Aided Techniques for Fading Estimation and Diversity Reception in Multipath Fading Channels
}

\author{
M. H. Ng and S. W. Cheung \\ Department of Electrical and Electronic Engineering \\ The University of Hong Kong \\ Hong Kong \\ Tel: +852 28592711 \\ Fax: +852 25598738 \\ Email: mhng@eee.hku.hk and swcheung@eee.hku.hk
}

Submitted to

Wireless Networks

22 November 1999

Key words: PSA, bandwidth efficiency, fading estimation, diversity reception, Rayleigh fading 


\section{ABSTRACT}

A novel pilot-symbol-aided (PSA) fading estimation technique that combines the estimates from a conventional PSA technique and a bandwidth-efficient PSA technique to achieve better performances is proposed for digital signals in multipath fading channels. The conventional technique has better performances at low signal-tonoise ratios (SNRs), while the bandwidth-efficient technique is superior at high SNRs. Monte Carlo computer simulation has been used to assess the effects of the proposed combining technique on the bit-error-rate (BER) performances of 16-ary quadratureamplitude-modulation (16QAM), with and without two-branch diversity reception, in a flat Rayleigh fading channel. Results have shown that the combining technique has the advantages of both of the conventional technique and the bandwidth-efficient technique and is more preferred for use with diversity reception.

\section{INTRODUCTION}

Multipath fading is one of the main problems in digital mobile communications because it severely degrades the bit-error-rate (BER) performance and frequently causes high error floors to the systems. The use of pilot-symbol-aided (PSA) transmission to combat multipath fading in the digital mobile communication systems has been widely studied [1-8]. At a PSA transmitter, known pilot symbols are periodically inserted into the data sequence for transmission. At the receiver, the pilot symbols are extracted and the fading effects on them are computed. The fading effects on the pilot symbols are then interpolated to form the estimates of the fading effects on the data symbols. Conventional PSA techniques employ solely the pilot symbols during the fading estimation process [1-5]. When the fading rate is fast or the pilot symbol rate is slow, these conventional techniques require the use of high order 
interpolating functions, or the estimates will be less accurate and high error floors occur [2,5]. However, high order interpolating functions require larger buffer space and more computational load, as well as longer delay which is of primary concern to voice services in mobile communications. If the fading effects have to be estimated more accurately using low order interpolating functions, the pilot symbols need to be sent more frequently than the theoretical rate of $f_{D} T_{F}=0.5$, where $f_{D}$ is the maximum Doppler frequency and $1 / T_{F}$ is the pilot symbol rate [3,4]. However, an increase in the pilot symbol rate means an increase in the bandwidth redundancy used to transmit the pilot symbols in the PSA systems. For example, in the Rayleigh fading channels with $f_{D} T=5 \times 10^{-3}$, where $T$ is the channel symbol period, researchers have suggested a frame length of $16[3,4]$, leading to $f_{D} T_{F}=0.08$ and a bandwidth redundancy of $6.25 \%$.

Recently, a fading estimation technique that employs both pilot and data symbols has been proposed to significantly reduce the bandwidth redundancy of the PSA systems [8]. With this technique, the initial estimates from the pilot symbols are adaptively refined using the detected data symbols, and linear interpolation is used to form the estimates for the remaining data symbols. In the Rayleigh fading channels with $f_{D} T=5 \times 10^{-3}$, this technique requires a frame length of 70 to provide satisfactory BER performances, leading to $f_{D} T_{F}=0.35$ and a bandwidth redundancy of $1.43 \%$ only. However, this bandwidth-efficient technique only works effectively at high signal-to-noise ratios (SNRs), but is inferior to the conventional techniques with the same order at low SNRs. In systems where diversity reception is employed to combat multipath fading [9-12], the operating SNRs are usually lower and so the fading estimation technique has to work effectively at lower SNRs, thus the bandwidth-efficient PSA technique becomes less attractive [8]. The performance of 
the bandwidth-efficient technique at low SNRs is degraded by noise included in the fading estimates obtained from the data symbols. Researchers have proposed different PSA techniques that also employ the data symbols for fading estimation [6,7]. These data-aided techniques use discriminators to select the feedback data and noisesmoothing filters to reduce the noise effects on the refined estimates. However, both of the discriminators and smoothing filters add computational load and delay to the systems.

In this paper, a novel PSA technique is proposed to combine the fading estimates from the bandwidth-efficient technique and a conventional technique. The fading estimates are first obtained separately with the bandwidth-efficient technique and the conventional technique, weighted and then summed according to their estimated SNRs. When diversity reception is employed, the signals from different branches are summed through maximal ratio combining [9]. Monte Carlo computer simulation has been used to assess the proposed technique on the BER performances of 16-ary quadrature-amplitude-modulation (16QAM) in the flat Rayleigh fading channels. Results have shown that the proposed technique requires very low bandwidth redundancies to provide satisfactory BER performances at low SNRs, and thus is suitable for use with diversity reception.

\section{SYSTEM MODEL}

The baseband equivalent block diagram of the land mobile communication system used in this paper is shown in Fig. 1. In the transmitter, a pilot symbol from a pseudo-random symbol sequence $\left\{p_{k, 0}\right\}$ is multiplexed with every $(L-1)$ data symbols $\left\{d_{k, i}\right\}$ to form a frame of $L$ symbols as shown in Fig. 2. A pseudo-random sequence of pilot symbols is used to avoid transmitting tones [1]. To minimize the fading 
estimation error due to additive white Gaussian noise (AWGN), the pilot symbols are chosen from the signal vectors with the highest energy levels in the 16QAM constellation [3]. The signal at the output of the pre-modulation filter is

$$
s(t)=\sum_{n} q_{n} a(t-n T)
$$

where $q_{n}$ is the $n$-th pilot or data symbol, $a(t)$ is the impulse response of the premodulation filter which has a square-root raised-cosine frequency response with a rolloff factor of $50 \%$.

The transmission path in Fig. 1 is a flat Rayleigh fading channel and is modeled as shown in Fig. 3, where the transmitted signal is multiplied by the fading signal [13]

$$
y(t)=y_{I}(t)+j y_{Q}(t)
$$

where $j=\sqrt{-1}$, and both $y_{I}(t)$ and $y_{Q}(t)$ are independent zero-mean Gaussian random processes, each with variance $\sigma^{2}=0.5$ and power spectrum [13]

$$
S(f)= \begin{cases}\frac{\sigma^{2}}{\pi \sqrt{f_{D}{ }^{2}-f^{2}}}, & |f| \leq f_{D} \\ 0, & |f|>f_{D}\end{cases}
$$

Stationary AWGN with one-sided power-spectral-density (PSD) of $N_{0}$ is assumed to be added at the input of the receiver.

At the receiver, the faded signal is filtered by the post-demodulation filter which has the same impulse response $a(t)$ as the pre-modulation filter to produce the signal

$$
r(t)=\sum_{n} q_{n} a(t-n T) y(t) \otimes a(t)+w(t)
$$

where $\otimes$ denotes the convolution process, $y(t)$ represents the fading effects, and $w(t)$ is the filtered noise waveform. The received signal is sampled in synchronism at time 
$t=n T$ seconds. Assume that the fading rate is slow compared to the symbol rate, so that the intersymbol interference (ISI) caused by the fading process can be neglected. Further assume that frame synchronization has been achieved and that $a(t) \otimes a(t)=1$ at time $t=0$. The received signal at the $i$-th position of the $k$-th frame is given by

$$
r_{k, i}=q_{k, i} y_{k, i}+w_{k, i}
$$

where $q_{k, i}$ is either a pilot symbol or a data symbol, and $y_{k, i}$ and $w_{k, i}$ are the fading effect and noise sample on the $i$-th symbol of the $k$-th frame, respectively.

Since the receiver has a prior knowledge of the pilot symbol sequence, the fading effects on the pilot symbols can be estimated and then used to estimate the fading effects on the data symbols. In the following section, the conventional technique [3] and the bandwidth-efficient technique [8] for fading estimation are first briefly described. Then a novel technique that combines both of the conventional technique and the bandwidth-efficient technique to achieve better estimation is proposed. Afterward, a diversity reception technique used to illustrate the advantages of the proposed technique is described.

\section{FADING ESTIMATION}

\section{A. Conventional Technique}

Here, the fading effects, $y_{k, 0}$ and $y_{k+1,0}$, on the pilot symbols, $p_{k, 0}$ and $p_{k+1,0}$, of the $k$-th and ( $k+1)$-th frames are estimated, respectively, using (5) as

$$
\hat{y}_{k, 0}=r_{k, 0} / p_{k, 0}=y_{k, 0}+w_{k, 0} / p_{k, 0}
$$

and

$$
\hat{y}_{k+1,0}=r_{k+1,0} / p_{k+1,0}=y_{k+1,0}+w_{k+1,0} / p_{k+1,0}
$$

The fading effect $y_{k, i}$ on the $i$-th data symbol $d_{k, i}$ of the $k$-th frame is then estimated using linear interpolation on $\hat{y}_{k, 0}$ and $\hat{y}_{k+1,0}$ as 


$$
\begin{aligned}
\hat{y}_{k, i} & =\frac{(L-i) \hat{y}_{k, 0}+i \times \hat{y}_{k+1,0}}{L}, \quad 0<i<L \\
& =\frac{(L-i)\left(y_{k, 0}+w_{k, 0} / p_{k, 0}\right)+i \times\left(y_{k+1,0}+w_{k+1,0} / p_{k+1,0}\right)}{L}
\end{aligned}
$$

which is used to produce the compensated symbol as

$$
\hat{r}_{k, i}=\frac{r_{k, i}}{\hat{y}_{k, i}}=\frac{L\left(y_{k, i} d_{k, i}+w_{k, i}\right)}{(L-i)\left(y_{k, 0}+w_{k, 0} / p_{k, 0}\right)+i \times\left(y_{k+1,0}+w_{k+1,0} / p_{k+1,0}\right)}
$$

\section{B. Bandwidth-Efficient Technique}

The square-error (SE) of the first data symbol $d_{k, 1}$ of the $k$-th frame can be calculated using (8) as

$$
\begin{aligned}
e_{k, 1} & =\left|\hat{r}_{k, 1}-d_{k, 1}\right|^{2} \\
& =\left.\left|\frac{L\left(y_{k, 1} d_{k, 1}+w_{k, 1}\right)-d_{k, 1}\left[(L-1)\left(y_{k, 0}+w_{k, 0} / p_{k, 0}\right)+y_{k+1,0}+w_{k+1,0} / p_{k+1,0}\right]}{(L-1)\left(y_{k, 0}+w_{k, 0} / p_{k, 0}\right)+y_{k+1,0}+w_{k+1,0} / p_{k+1,0}}\right|\right|^{2} \\
& =\left|\frac{d_{k, 1}\left(L y_{k, 1}-L y_{k, 0}+y_{k, 0}-y_{k+1,0}\right)+L w_{k, 1}-(L-1) d_{k, 1} w_{k, 0} / p_{k, 0}-d_{k, 1} w_{k+1,0} / p_{k+1,0}}{L y_{k, 0}-y_{k, 0}+y_{k+1,0}+(L-1) w_{k, 0} / p_{k, 0}+w_{k+1,0} / p_{k+1,0}}\right|^{2}
\end{aligned}
$$

Denoting $\quad \bar{y}_{k, i}=\frac{(L-i) y_{k, 0}+i \times y_{k+i, 0}}{L}, \quad 0<i<L$

which are the fading estimates obtained by using linear interpolation on $y_{k, 0}$ and $y_{k+1,0}$ in the absence of noise. Then the SE in (9) can be written as

$e_{k, 1}=\left|\frac{d_{k, 1} L\left(y_{k, 1}-\bar{y}_{k, 1}\right)+L w_{k, 1}-(L-1) d_{k, 1} w_{k, 0} / p_{k, 0}-d_{k, 1} w_{k+1,0} / p_{k+1,0}}{L \bar{y}_{k, 1}+(L-1) w_{k, 0} / p_{k, 0}+w_{k+1,0} / p_{k+1,0}}\right|^{2}$

Similarly, the SE of the last data symbol $d_{k, L-1}$ of the same frame can be obtained as 
$e_{k, L-1}=\left|\frac{d_{k, L-1} L\left(y_{k, L-1}-\bar{y}_{k, L-1}\right)+L w_{k, L-1}-d_{k, L-1} w_{k, 0} / p_{k, 0}-(L-1) d_{k, L-1} w_{k+1,0} / p_{k+1,0}}{L \bar{y}_{k, L-1}+w_{k, 0} / p_{k, 0}+(L-1) w_{k+1,0} / p_{k+1,0}}\right|^{2}$

The terms $\left(y_{k, 1}-\bar{y}_{k, 1}\right)$ and $\left(y_{k, L-1}-\bar{y}_{k, L-1}\right)$ in (11a) and (11b), respectively, represent the errors due to linear interpolation and, in a slow fading environment, have about the same value. At high SNRs, the noise terms in (11a) and (11b) can be neglected and so the difference between (11a) and (11b) is only the terms $\bar{y}_{k, 1}$ and $\bar{y}_{k, L-1}$ in the denominators. The larger is this term, the smaller is the resultant SE of the data symbol. Based on these findings, the bandwidth-efficient technique [8] was proposed to minimize the SE by selecting the equation, (11a) or (11b), with the larger interpolated value, $\bar{y}_{k, 1}$ or $\bar{y}_{k, L-1}$.

The bandwidth-efficient technique starts with the fading estimates $\hat{y}_{k, 0}$ and $\hat{y}_{k+1,0}$ using (6a) and (6b), respectively. The magnitudes $\left|\hat{y}_{k, 0}\right|^{2}$ and $\left|\hat{y}_{k+1,0}\right|^{2}$ are calculated and the larger one is selected. Linear interpolation is then used to form an initial estimate of the fading effect, $y_{k, 1}$ or $y_{k, L-1}$, that is adjacent to the larger one of $\left|\hat{y}_{k, 0}\right|^{2}$ or $\left|\hat{y}_{k+1,0}\right|^{2}$. If $\left|\hat{y}_{k, 0}\right|^{2}$ is larger than $\left|\hat{y}_{k+1,0}\right|^{2}$, then $\left|\hat{y}_{k, 1}\right|^{2}$ will be larger than $\left|\hat{y}_{k, L-1}\right|^{2}$ and thus $\hat{y}_{k, 1}$ is computed using (7), otherwise $\hat{y}_{k, L-1}$ is computed instead. Without loss of generality, we assume that $\left|\hat{y}_{k, 0}\right|^{2}$ is larger and thus $\hat{y}_{k, 1}$ is computed. The estimate $\hat{y}_{k, 1}$ is used to obtain the compensated symbol $\hat{r}_{k, 1}$ using (8). The symbol $\hat{r}_{k, 1}$ is then threshold detected to give the detected symbol $\hat{d}_{k, 1}$ that is a possible vector in the 16QAM signal constellation. The symbol $\hat{d}_{k, 1}$ is used to obtain a new estimate of $y_{k, 1}$ as 


$$
\tilde{y}_{k, 1}=r_{k, 1} / \hat{d}_{k, 1}
$$

The process repeats but now with $\hat{y}_{k, 0}$ replaced by $\tilde{y}_{k, 1}$, and for the estimation of $y_{k, 2}$ or $y_{k, L-1}$, depending on which of $\left|\tilde{y}_{k, 1}\right|^{2}$ or $\left|\hat{y}_{k+1,0}\right|^{2}$ is larger. Since $y_{k, 1}$ has a stronger time correlation with both $y_{k, 2}$ and $y_{k, L-1}$ than $y_{k, 0}$ has, and the interpolating length is reduced from $L$ to $(L-1)$, the error due to linear interpolation should be reduced and so a more accurate estimate of $y_{k, 2}$ or $y_{k, L-1}$ should be obtained, provided that $d_{k, 1}$ has been correctly detected. To summarize the process, the fading effect $y_{k, i}$ is estimated by employing both of the pilot and data symbols as

$$
\begin{aligned}
\tilde{y}_{k, i} & =\frac{(i-m) \tilde{y}_{k, i+1}+\tilde{y}_{k, m}}{i-m+1} \\
\text { or } \quad \tilde{y}_{k, i} & =\frac{(n-i) \tilde{y}_{k, i-1}+\tilde{y}_{k, n}}{n-i+1}
\end{aligned}
$$

where $\tilde{y}_{k, m}$ and $\tilde{y}_{k, n}$ are the fading estimates on the $m$-th and $n$-th symbols, respectively, and $m<i<n$.

\section{Combining Technique}

The two fading estimates, $\hat{y}_{k, i}$ and $\tilde{y}_{k, i}$, obtained using the conventional technique in (7) and the bandwidth-efficient fading estimation technique in (13), respectively, are used to compensate for the fading effect on the received symbol $r_{k, i}$ to give the respective compensated symbols

$$
\hat{r}_{k, i}=r_{k, i} / \hat{y}_{k, i}
$$

and

$$
\tilde{r}_{k, i}=r_{k, i} / \tilde{y}_{k, i}
$$


Let $\hat{d}_{k, i}$ and $\tilde{d}_{k, i}$ be the vectors closest to $\hat{r}_{k, i}$ and $\tilde{r}_{k, i}$, respectively, in the 16QAM signal constellation. The respective SNRs of $\hat{r}_{k, i}$ and $\tilde{r}_{k, i}$ can be estimated as

$$
\begin{aligned}
\hat{z}_{k, i} & =\left|\hat{y}_{k, i} \hat{d}_{k, i}\right|^{2} /\left|r_{k, i}-\hat{y}_{k, i} \hat{d}_{k, i}\right|^{2} \\
\text { and } & \tilde{z}_{k, i}=\left|\tilde{y}_{k, i} \tilde{d}_{k, i}\right|^{2} /\left|r_{k, i}-\tilde{y}_{k, i} \tilde{d}_{k, i}\right|^{2}
\end{aligned}
$$

Here, we propose to combine $\hat{y}_{k, i}$ and $\tilde{y}_{k, i}$, according to $\hat{z}_{k, i}$ and $\tilde{z}_{k, i}$, as

$$
y_{k, i}^{\prime}=\frac{\hat{y}_{k, i} \hat{z}_{k, i}+\tilde{y}_{k, i}\left(\tilde{z}_{k, i}\right)^{x}}{\hat{z}_{k, i}+\left(\tilde{z}_{k, i}\right)^{x}}
$$

where the power $x$ is an arbitrary number to be optimized. The rationale behind (16) is explained as follows. Since the conventional technique performs better than the bandwidth-efficient technique at low SNRs, while the bandwidth-efficient technique is superior at high SNRs [8], the SNRs $\hat{z}_{k, i}$ and $\widetilde{z}_{k, i}$ can be used as weighting factors to combine the fading estimates $\hat{y}_{k, i}$ and $\tilde{y}_{k, i}$ together. The combining process in (16), in fact, accentuates the fading estimate with the higher SNR and suppresses the one with the lower SNR. Note that when $\hat{z}_{k, i}$ is equal to $\widetilde{z}_{k, i}$ and the value of $x$ is set to 1 , the combined estimate is simply the average of the two fading estimates. When the value of $x$ is increased, we place more weight on the estimate obtained using the bandwidth-efficient technique.

The combined estimate $y_{k, i}^{\prime}$ is used to give the compensated symbol as

$$
r_{k, i}^{\prime}=r_{k, i} / y_{k, i}^{\prime}
$$

which is then threshold detected to give the output symbol $d_{k, i}^{\prime}$. The symbol $d_{k, i}^{\prime}$ is used to provide a new estimate of $y_{k, i}$ as

$$
\tilde{y}_{k, i}=r_{k, i} / d_{k, i}^{\prime}
$$


which is then used as $\tilde{y}_{k, i+1}, \tilde{y}_{k, m}, \tilde{y}_{k, i-1}$ or $\tilde{y}_{k, n}$ in (13) to estimate the fading effect on the next data symbol of the same frame.

\section{Diversity Reception}

To illustrate the advantages of using the combining technique in the diversity reception systems, we adopt the maximal ratio combining technique used in [9] where the compensated signals $\left\{r_{h, k, i}^{\prime}\right\}$ from the $h$ different branches are weighted and summed to give the $i$-th combined symbol of the $k$-th frame as [9]

$$
c_{k, i}^{\prime}=\sum_{h} r_{h, k, i}^{\prime}\left|y_{h, k, i}^{\prime}\right|^{2} / \sum_{h}\left|y_{h, k, i}^{\prime}\right|^{2}
$$

where $\left|y_{h, k, i}^{\prime}\right|^{2}$ is the estimated power of the fading effect. The signal $c_{k, i}^{\prime}$ in (19) is then threshold detected to give the output symbol $d_{k, i}^{\prime}$. This whole process continues until all data symbols within the received signal have been detected.

\section{RESULTS AND DISCUSSIONS}

Monte Carlo computer simulation has been carried out to assess the proposed combining technique with different values of $x$, with and without using two-branch diversity reception in (19), on the BER performances of the PSA-16QAM system shown in Fig. 1, where the transmission path is a flat Rayleigh fading channel with a normalized fading rate of $f_{D} T=5 \times 10^{-3}$. The effects of using the combining technique with different values of $x$ on the BER performances of the system have been studied. In all tests, the SNR is taken as

$$
S N R=E_{b} / N_{o}=\frac{E_{d} \times(L-1)+E_{p}}{M \times(L-1) \times N_{o}}
$$


where $E_{d}$ and $E_{p}$ are the average transmitted energies per data symbol and per pilot symbol, respectively, $M$ is the number of information bits carried per data symbol, and $N_{0}$ is the one-sided noise-PSD. For 16QAM, $M=4, E_{d}=10$ and $E_{p}=18$, and hence

$$
S N R=\frac{2.5+4.5 /(L-1)}{N_{o}}
$$

With the normalized fading rate of $f_{D} T=5 \times 10^{-3}$ and no diversity reception, the results of simulation on the BER performances of the PSA-16QAM system, using the conventional technique, the bandwidth-efficient technique and the combining technique with $x=1,2,3$ and 4, and with $L=100,75$ and 50 are shown in Figs. 4a, 4b and 4c, respectively. Note that with $L=100,75$ and 50, the system achieves $f_{D} T_{F}$ $=0.5,0.35$ and 0.25 , respectively, leading to the corresponding bandwidth redundancies of only $1 \%, 1.43 \%$ and $2 \%$. It can be seen in Fig. 4 that with such low bandwidth redundancies, the conventional technique has very high error floors and cannot achieve a BER of $10^{-3}$ which is the minimum BER required for voice services of the UMTS [14] and IS-54 [15]. The bandwidth-efficient technique appears to have no error floor in the SNRs tested and can achieve a BER of $10^{-3}$ at higher SNRs. However, at low SNRs, the bandwidth-efficient technique is inferior to the conventional technique. Thus in the diversity reception systems where the operating SNRs are relatively lower and the required pre-diversity BER should also be low enough for use with diversity reception to achieve a post-diversity BER of $10^{-3}$, both of the conventional technique and the bandwidth-efficient technique may not be appropriate. Figure 4 shows that, by adjusting the value of $x$, the combining technique can have the advantages of both of the conventional technique and the bandwidthefficient technique (i.e. the superior BER performances of using the bandwidth- 
efficient technique at high SNRs and of using the conventional technique at low SNRs can be obtained at the same time.) As $x$ is increased from 1 to 4, it can be seen that the BERs at high SNRs become closer to those using the bandwidth-efficient technique, yet the BERs at low SNRs remain similar to those using the conventional technique. Thus we have the freedom to select the type of BER performances by using the value of $x$. With $x=1$, the combined estimate is simply the average of the estimates obtained from the conventional technique and the bandwidth-efficient technique, and the BERs at high SNRs are substantially reduced relative to those of using the conventional technique. With $x$ increased to 2, the reduction in BERs is also significant at high SNRs. However, the reduction becomes less significant for further increase of $x$. With $L=70$, Fig. 4b shows that the conventional technique has an error floor at the BER of $5 \times 10^{-2}$, while the bandwidth-efficient technique has no error floor under the SNRs tested and reduces the BER to less than $2 \times 10^{-4}$ at SNR $=40$ dB. But, the bandwidth-efficient technique is only superior to the conventional technique when the SNRs is above $17.5 \mathrm{~dB}$. When $x=1$ is used, the combining technique reduces the BER from $5 \times 10^{-2}$ to $5 \times 10^{-3}$ at $\mathrm{SNR}=40 \mathrm{~dB}$. With $x$ increased to 2, the BER is reduced to $5 \times 10^{-4}$. However, when $x$ is further increased to 3 and 4, the BERs are only reduced to $3 \times 10^{-4}$ and $2 \times 10^{-4}$, respectively. Comparing the results in Figs. 4a and 4c indicates that, with $L=50$, the combining technique with $x=4$ outperforms the one with $x=1$ only at SNRs above $22 \mathrm{~dB}$; whereas with $L=100$, the combining technique with $x=4$ outperforms the one with $x$ $=1$ at SNRs above $12 \mathrm{~dB}$. This is because the bandwidth-efficient technique is more advantageous with longer frame lengths and thus more weight should be placed on the estimate from the bandwidth-efficient technique by using a larger $x$; thus when a larger frame length is used, a larger $\mathrm{x}$ is preferred. 
With two-branch diversity reception, the BER performances of the system with $L=100,70$ and 50 are shown in Fig. 5 . It can be seen that with such low bandwidth redundancies, even with two-branch diversity reception, the conventional technique still cannot achieve a BER of $10^{-3}$ in the SNRs tested. This is because the conventional technique cannot provide a pre-diversity BER low enough for diversity reception to achieve a BER of $10^{-3}$, as can be seen in Fig. 4. With $L=100$, Fig. 5a shows that the bandwidth-efficient technique can achieve a BER of $10^{-3}$ at $\mathrm{SNR}=$ $26.5 \mathrm{~dB}$, requiring $1 \mathrm{~dB}$ less than that using the combining technique with $x=4$. However, at the BER of $10^{-2}$, the combining technique with $x=4$ provides an advantage of $1 \mathrm{~dB}$ at SNR $=20 \mathrm{~dB}$ over the bandwidth-efficient technique. With $L=$ 50, Fig. 5c shows that the combining technique with $x=1$ can achieve a BER of $10^{-3}$ at $\mathrm{SNR}=19.5 \mathrm{~dB}$, leading to an advantage of $4.5 \mathrm{~dB}$ over the bandwidth-efficient technique. Clearly, in the diversity reception systems where the operating SNRs are relatively lower, the combining technique is more preferred.

With $L=70$, Fig. 5b shows that the bandwidth-efficient technique can achieve a BER of $10^{-3}$ at SNR $=24.5 \mathrm{~dB}$. Comparing this to the results without diversity reception in Fig. 4b indicates that diversity reception provides a gain of $8 \mathrm{~dB}$ at the BER of $10^{-3}$. However, it can be seen that the combining technique with $x=3$ can achieve a BER of $10^{-3}$ at SNR $=24 \mathrm{~dB}$, having a diversity gain of $10 \mathrm{~dB}$ when compared to the results in Fig. 4b. This can also be seen when we compare the results in Figs. $5 \mathrm{c}$ and $4 \mathrm{c}$, which show that at the BER of $10^{-3}$, the bandwidth-efficient technique has a diversity gain of only $8.5 \mathrm{~dB}$ whereas the combining technique with $\mathrm{x}$ $=3$ has a diversity gain of $11 \mathrm{~dB}$. These results indicate that the diversity reception systems can work more effectively with the combining technique. 
One of the main advantages of the proposed technique is that it provides the mobile system designers with more freedoms to select the more appropriate ranges of BERs for the mobile systems to operate by using a suitable value of $x$. Moreover, compared with the other data-aided techniques [6,7], the combining technique does not require the use of a noise-smoothing filter or a discriminator, and so it has little delay and requires simple computation.

\section{CONCLUSIONS}

A novel PSA technique that combines a conventional PSA technique and a bandwidth-efficient PSA technique for fading estimation in multipath fading channels has been proposed. The combining process is based on the SNRs of the fading estimates obtained from the two techniques. Computer simulation tests have been carried out on the BER performances of 16QAM in a Rayleigh fading channel using the combining technique. Results have shown that the combining technique has the advantages of both of the conventional technique and the bandwidth-efficient technique, i.e., the superior BER performances of using the bandwidth-efficient technique at high SNRs and of using the conventional technique at low SNRs can be obtained at the same time. By adjusting the value of $x$ in the combining technique, the mobile system designers can have more freedom to select the appropriate ranges of BERs for the mobile systems. Results of computer simulation on a two-branch diversity reception system have also shown that the combining technique is more preferred to the conventional technique and the bandwidth-efficient technique. With two-branch diversity reception and $f_{D} T=5 \times 10^{-3}$, the combining technique requires only a $2 \%$ bandwidth redundancy and a SNR of $19.5 \mathrm{~dB}$ to achieve the minimum required BER of $10^{-3}$ for voice services of the UMTS and IS-54. 


\section{REFERENCES}

[1] M. L. Moher and J. H. Lodge, “TCMP-A modulation and coding strategy for Rician fading channels,” IEEE J. Select. Areas Commun., vol. 7, no. 9, pp. 1347-1355, Dec. 1989.

[2] J. K. Cavers, “An analysis of pilot symbol assisted modulation for Rayleigh fading channels,” IEEE Trans. Veh. Technol., vol. 40, no. 4, pp. 686-693, Nov. 1991.

[3] S. Sampei and T. Sunaga, "Rayleigh fading compensation for QAM in land mobile radio communications,” IEEE Trans. Veh. Technol., vol. 42, no. 2, pp. 137-147, May 1993.

[4] C. L. Liu and K. Feher, "Pilot-symbol aided coherent M-ary PSK in frequency-selective fast Rayleigh fading channels,” IEEE Trans. Commun., vol. 42, no. 1, pp. 54-62, Jan. 1994.

[5] H. K. Lau and S. W. Cheung, "A pilot symbol-aided technique used for digital signal in multipath environments," in Proc. IEEE Int'l Conf. Commun., New Orleans, USA, May 1994, pp. 1126-1130.

[6] G. T. Irvine and P. J. McLane, "Symbol-aided plus decision-directed reception for PSK/TCM modulation on shadowed mobile satellite fading channels," IEEE J. Select. Areas Commun., vol. 10, no. 8, pp. 1289-1299, Oct. 1992.

[7] Y. Liu and S. D. Blostein, "Identification of frequency non-selective fading channels using decision feedback and adaptive linear prediction,” IEEE Trans. Commun., vol. 43, no. 2/3/4, pp. 1484-1492, Feb./Mar./Apr. 1995.

[8] M. H. Ng and S. W. Cheung, "Bandwidth-efficient pilot symbol aided technique,” Electronics Letters, vol. 34, no. 16, pp. 1548-1550, Aug. 1998.

[9] T. Sunaga and S. Sampei, "Performance of multi-level QAM with postdetection maximal ratio combining space diversity for digital land-mobile radio communications,” IEEE Trans. Veh. Technol., vol. 42, no. 3, pp. 294301, Aug. 1993.

[10] M. Sawahashi and F. Adachi, "Multicarrier 16QAM transmission with diversity reception,” Electronics Letters, vol. 32, no. 6, pp. 522-523, Mar. 1996.

[11] J. Lu, T. T. Tjhung, and C. C. Chai, "Error probability performance of Lbranch diversity reception of MQAM in Rayleigh fading," IEEE Trans. Commun., vol. 46, no. 2, pp. 179-181, Feb. 1998. 
[12] P. Schramm and R. R. Muller, "Pilot symbol assisted BPSK on Rayleigh fading channels with diversity: performance analysis and parameter optimization,” IEEE Trans. Commun., vol. 46, no. 12, pp. 1560-1563, Dec. 1998.

[13] C. Loo and N. Secord, "Computer models for fading channels with applications to digital transmission,” IEEE Trans. Veh. Technol., vol. 40, pp. 700-707, Nov. 1991.

[14] Z. Zvonar, P. Jung, and K. Kammerlander, (eds.), GSM: Evolution towards 3rd Generation Systems, Boston: Kluwer, 1999.

[15] H. V. Poor and G. W. Wornell, (eds.), Wireless Communications: Signal Processing Perspectives, New Jersey: Prentice Hall, 1998.

\section{FIGURES}

Fig. 1 System block diagram

Fig. 2 Frame structure

Fig. 3 Rayleigh fading model

Fig. 4 BER performances of 16QAM, without diversity reception and with $f_{D} T=5 \times 10^{-3}$, using conventional technique, bandwidth-efficient technique and proposed technique with $x=1,2,3$ and 4, and with (a) $L=100$, (b) $L=70$ and (c) $L=50$.

Fig. 5 BER performances of 16QAM, with 2-branch diversity reception and $f_{D} T=5 \times 10^{-3}$, using conventional technique, bandwidth-efficient technique and proposed technique with $x=1,2,3$ and 4, and with (a) $L=100$, (b) $L=70$ and (c) $L=50$. 


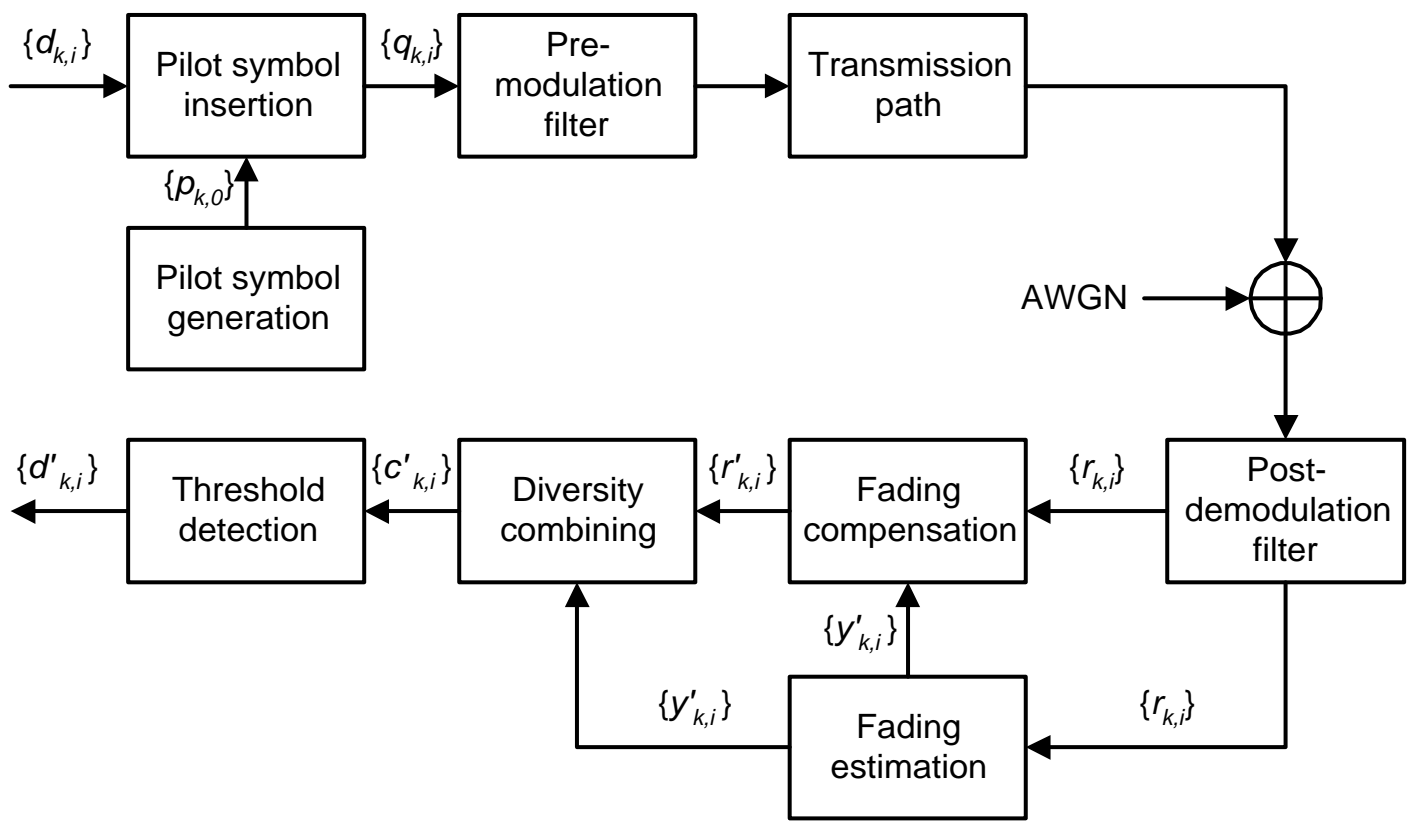

Fig. 1 System block diagram

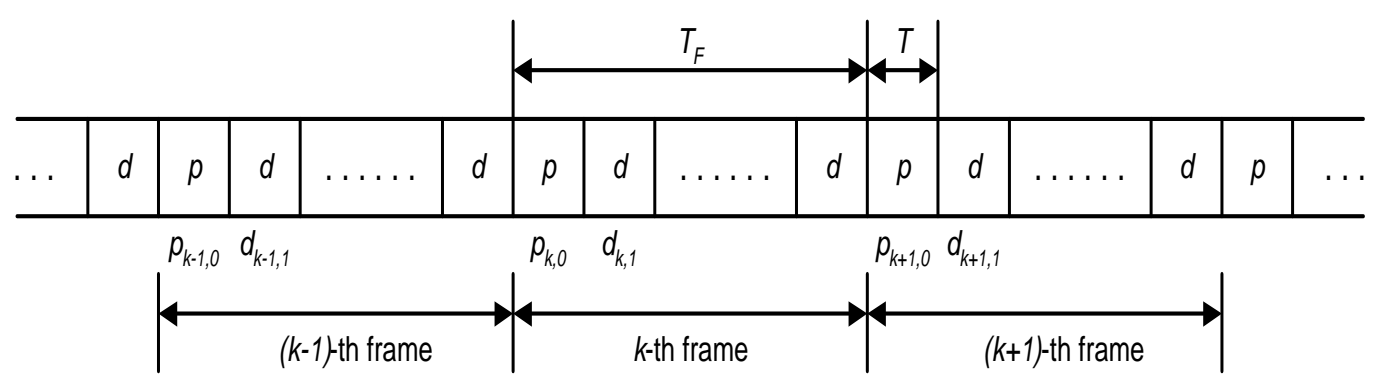

$p$ : pilot symbol

$d$ : data symbol

Fig. 2 Frame structure

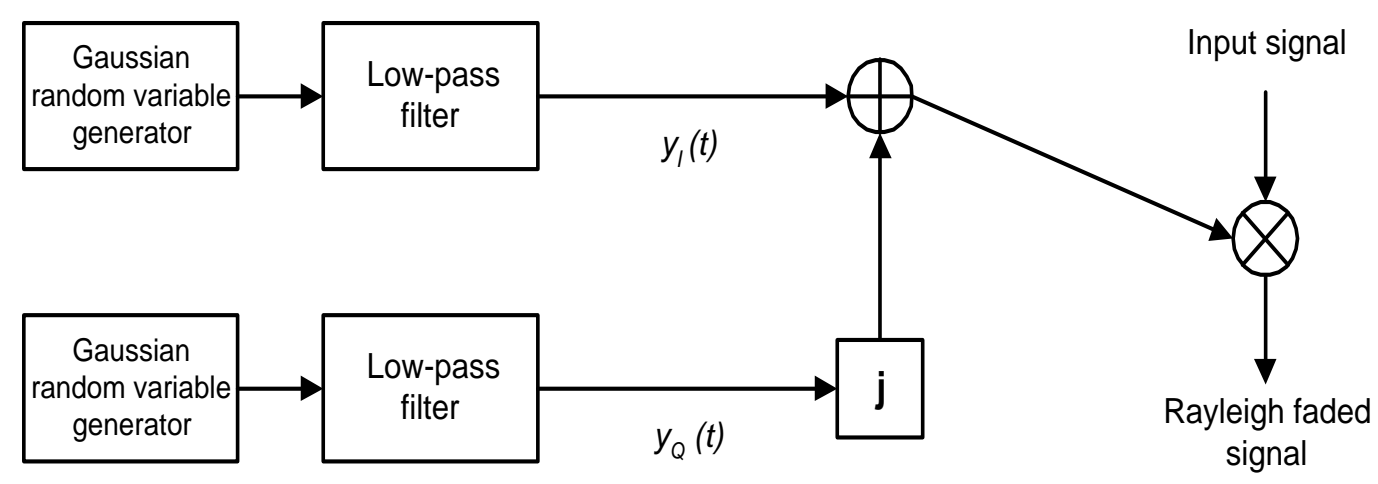

Fig. 3 Rayleigh fading model 


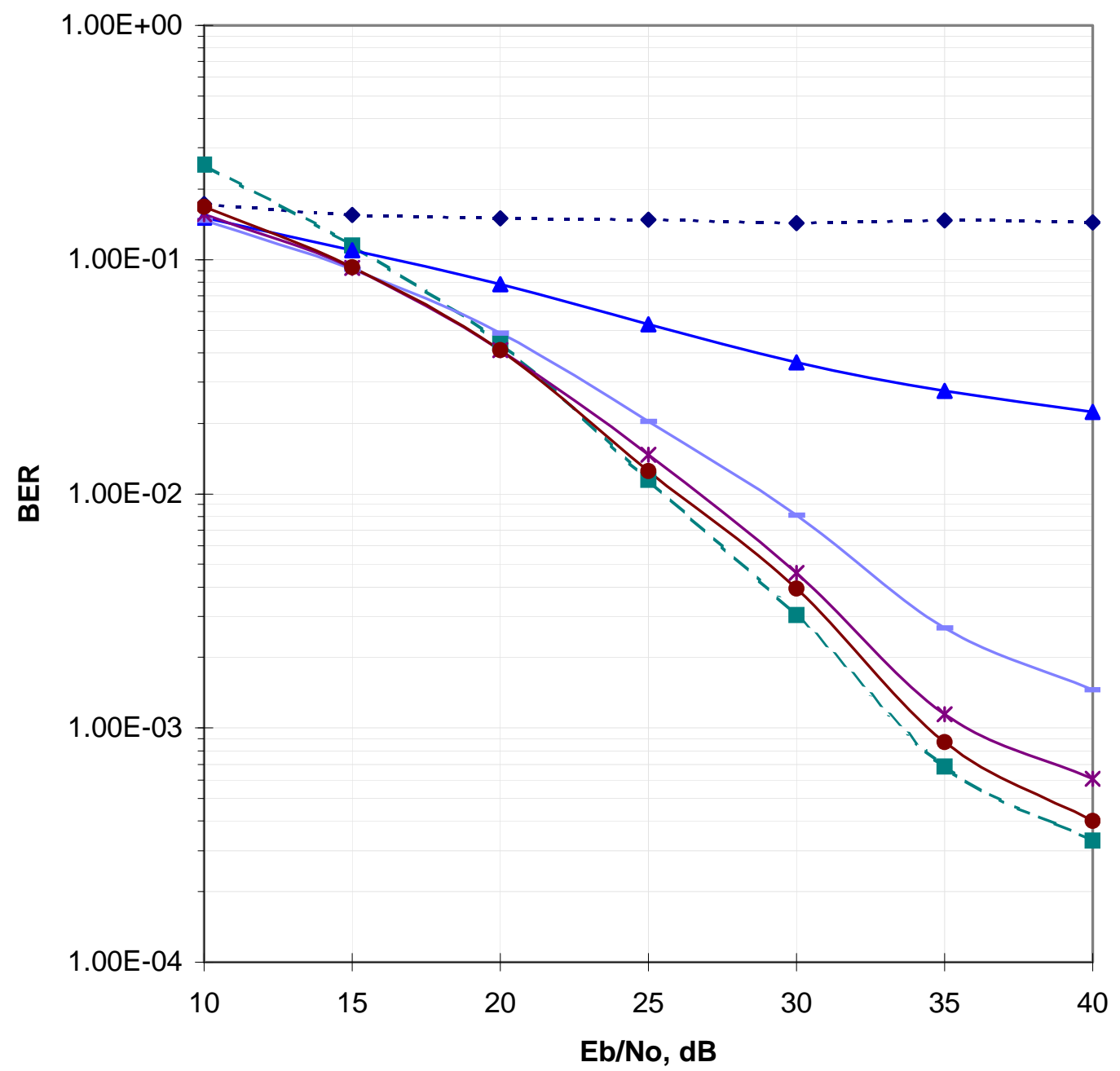

$\begin{array}{ll}-- \text { conventional, } L=100 & -- \text { bandwidth-efficient, } \mathrm{L}=100 \\ \longrightarrow \text { proposed, } x=1, L=100 & - \text { proposed, } x=2, L=100 \\ \longrightarrow \text { proposed, } x=3, L=100 & \longrightarrow \text { proposed, } x=4, L=100\end{array}$

Fig. 4a 


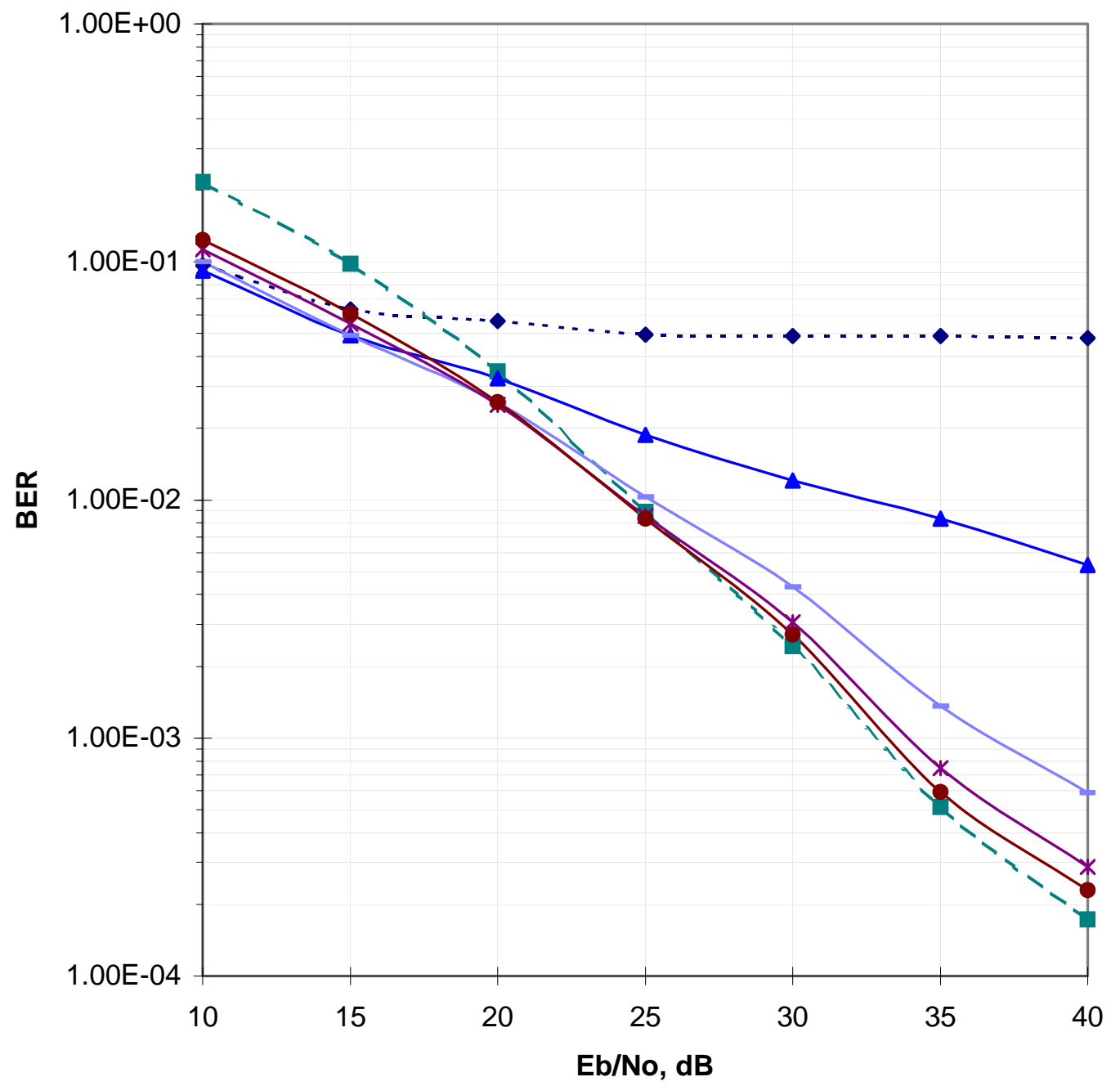

\begin{tabular}{ll|}
$-\sim-$ conventional, $L=70$ & -- bandwidth-efficient, $L=70$ \\
$\longrightarrow$ proposed, $x=1, L=70$ & proposed, $x=2, L=70$ \\
$\longrightarrow$ proposed, $x=3, L=70$ & $\longrightarrow$ proposed, $x=4, L=70$
\end{tabular}

Fig. 4b 


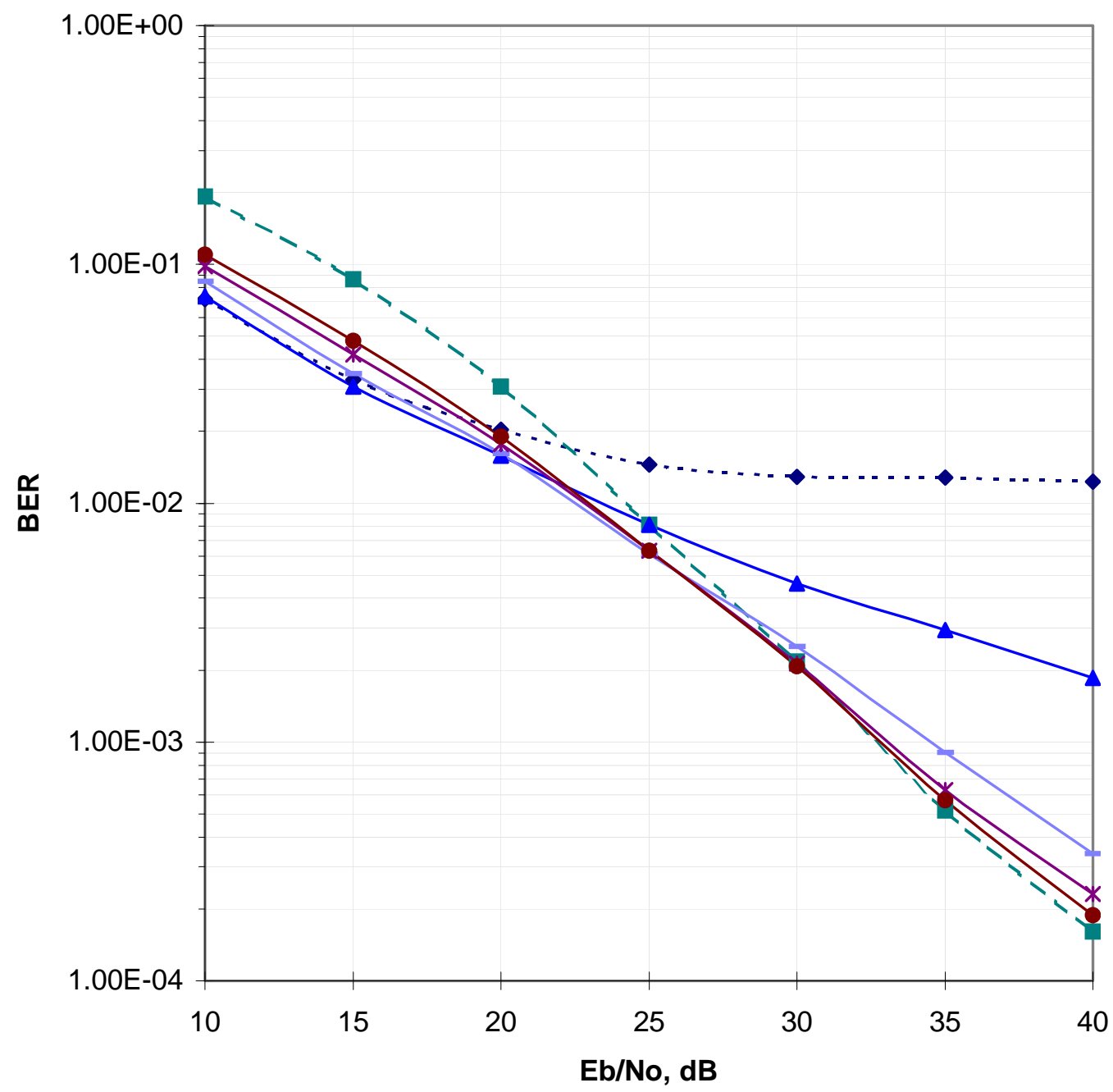

\begin{tabular}{ll|}
$-\rightarrow-$ conventional, $L=50$ & -- bandwidth-efficient, $L=50$ \\
$\longrightarrow$ proposed, $x=1, L=50$ & proposed, $x=2, L=50$ \\
$\longrightarrow$ proposed, $x=3, L=50$ & $\longrightarrow$ proposed, $x=4, L=50$
\end{tabular}

Fig. 4c

Fig. 4 BER performances of 16QAM, without diversity reception and with $f_{D} T=5 \times 10^{-3}$, using conventional technique, bandwidth-efficient technique and proposed technique with $x=1,2,3$ and 4, and with (a) $L=100$, (b) $L=70$ and (c) $L=50$. 


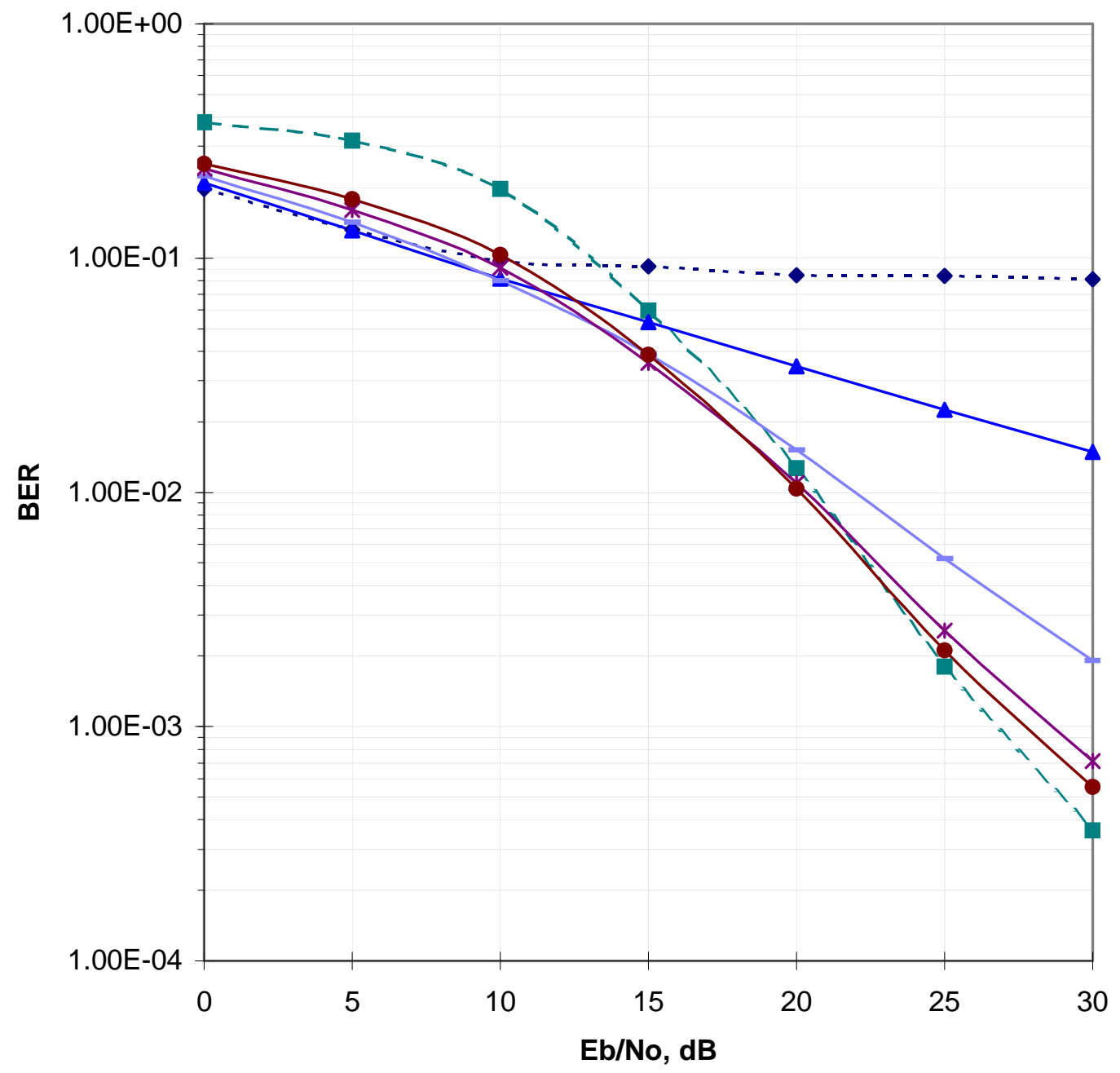

$\begin{array}{ll}-\cdots \text { - conventional, } L=100 & -- \text { bandwidth-efficient, } L=100 \\ \longrightarrow \text { proposed, } x=1, L=100 & - \text { proposed, } x=2, L=100 \\ \longrightarrow \text { - proposed, } x=3, L=100 & \longrightarrow \text { proposed, } x=4, L=100\end{array}$

Fig. 5a 


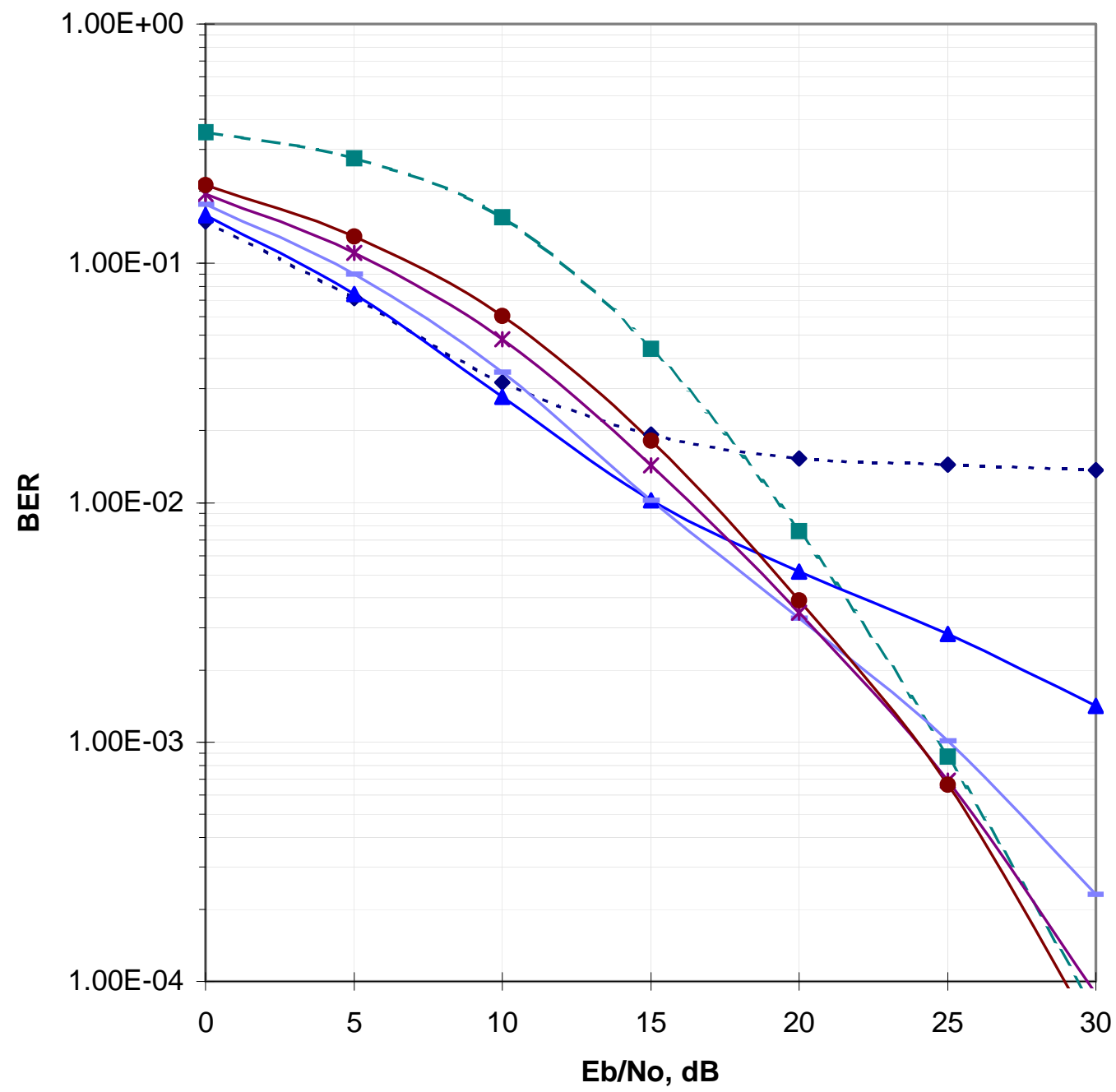

$\begin{array}{ll}\cdots-\text { conventional, } L=70 & -- \text { bandwidth-efficient, } \mathrm{L}=70 \\ \longrightarrow \text { proposed, } x=1, \mathrm{~L}=70 & - \text { proposed, } x=2, \mathrm{~L}=70 \\ \longrightarrow \text { proposed, } x=3, \mathrm{~L}=70 & \longrightarrow \text { proposed, } \mathrm{x}=4, \mathrm{~L}=70\end{array}$

Fig. 5b 


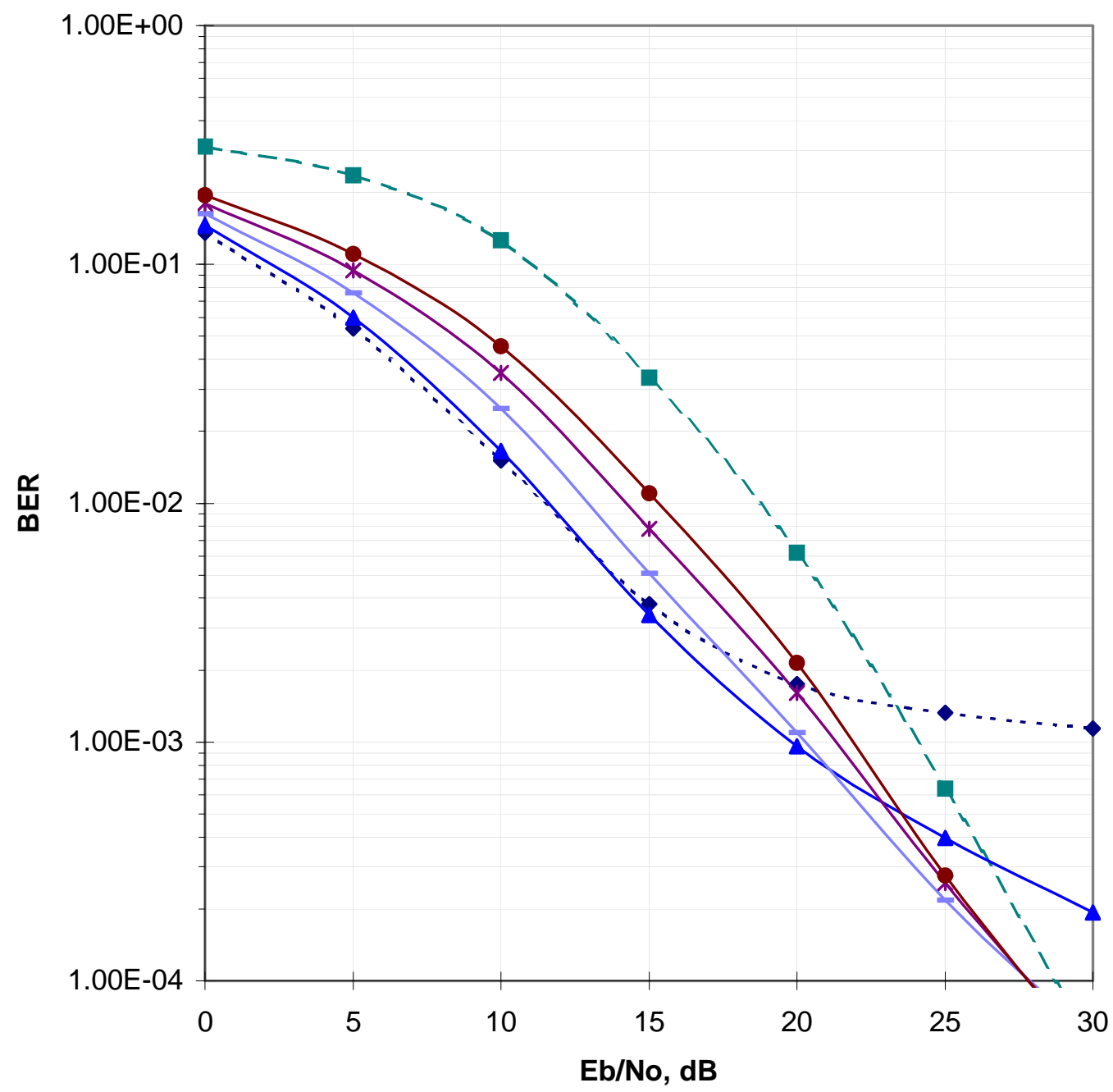

$\begin{array}{ll}\cdots-\text { conventional, } L=50 & -- \text { bandwidth-efficient, } L=50 \\ \longrightarrow \text { proposed, } x=1, L=50 & - \text { proposed, } x=2, L=50 \\ \longrightarrow \text { proposed, } x=3, L=50 & \longrightarrow \text { proposed, } x=4, L=50\end{array}$

Fig. 5c

Fig. 5 BER performances of 16QAM, with 2-branch diversity reception and $f_{D} T=5 \times 10^{-3}$, using conventional technique, bandwidth-efficient technique and proposed technique with $x=1,2,3$ and 4, and with (a) $L=100$, (b) $L=70$ and (c) $L=50$. 